\title{
Clinical effectiveness of the START (STrAtegies for RelaTives) psychological intervention for family carers and the effects on the cost of care for people with dementia: 6-year follow-up of a randomised controlled trial
}

Gill Livingston, Monica Manela, Aidan O'Keeffe, Penny Rapaport, Claudia Cooper, Martin Knapp, Derek King, Renee Romeo, Zuzana Walker, Juanita Hoe, Cath Mummery and Julie Barber

\section{Background}

The START (STrAtegies for RelaTives) intervention reduced depressive and anxiety symptoms of family carers of relatives with dementia at home over 2 years and was cost-effective.

\section{Aims}

To assess the clinical effectiveness over 6 years and the impact on costs and care home admission.

\section{Method}

We conducted a randomised, parallel group, superiority trial recruiting from 4 November 2009 to 8 June 2011 with 6-year follow-up (trial registration: ISCTRN 70017938). A total of 260 selfidentified family carers of people with dementia were randomised 2:1 to START, an eight-session manual-based coping intervention delivered by supervised psychology graduates, or to treatment as usual (TAU). The primary outcome was affective symptoms (Hospital Anxiety and Depression Scale, total score (HADS-T)). Secondary outcomes included patient and carer service costs and care home admission.

\section{Results}

In total, 222 (85.4\%) of 173 carers randomised to START and 87 to TAU were included in the 6-year clinical efficacy analysis. Over 72 months, compared with TAU, the intervention group had improved scores on HADS-T (adjusted mean difference -2.00 points, $95 \% \mathrm{Cl}-3.38$ to -0.63 ). Patient-related costs (START versus TAU, respectively: median $£ 5759 \mathrm{~V}$. $£ 16964$ in the final year; $P=0.07$ ) and carer-related costs (median $£ 377 v$. $£ 274$ in the final year) were not significantly different between groups nor were group differences in time until care home (intensity ratio START:TAU was $0.88,95 \% \mathrm{Cl} 0.58-1.35)$.

\section{Conclusions}

START is clinically effective and this effect lasts for 6 years without increasing costs. This is the first intervention with such a long-term clinical and possible economic benefit and has potential to make a difference to individual carers.

\section{Declarations of interest}

G.L., Z.W. and C.C. are supported by the UCLH National Institute for Health Research (NIHR) Biomedical Research Centre. G.L. and P.R. were in part supported by the National Institute for Health Research (NIHR) Collaboration for Leadership in Applied Health Research and Care (CLAHRC) North Thames at Bart's Health NHS Trust. The views expressed are those of the author(s) and not necessarily those of the NHS, the NIHR or the Department of Health. Z.W. reports during the conduct of the study; personal fees from GE Healthcare, grants from GE Healthcare, grants from Lundbeck, other from GE Healthcare, outside the submitted work.

\section{Keywords}

Family carer; randomised controlled trial; depression; dementia; cost.

\section{Copyright and usage}

(c) The Royal College of Psychiatrists 2019.
Families provide most of the care to people with dementia living at home. Family carers have worse physical health, more absences from work, lower quality of life and are more likely to be anxious or have depression than non-carers. ${ }^{1-4}$ Currently around 50 million people globally have dementia, projected to nearly triple by 2050 , and the present annual global cost is US $\$ 818$ billion. ${ }^{5}$ Nearly $85 \%$ of costs are family and social rather than medical costs. ${ }^{6}$

The START (STrAtegies for RelaTives) multicomponent intervention for family carers is individually delivered by supervised psychology graduates (with a first degree in psychology and no clinical training) and was tested by our research team in a randomised controlled trial (RCT). It was the first trial to show both clinical effectiveness (reduced anxiety and depressive symptoms, decreased depression 'caseness', improved quality of life) and cost-effectiveness for family carers of people with dementia. ${ }^{7,8}$ Methods and results up to 2 years of follow-up have been reported in detail elsewhere. ${ }^{7-11}$ We found that START carers had a decrease in symptom score that was greater than the minimum clinically important difference and at 8 months were one-fifth as likely to have case-level depression. These benefits persisted for 2 years, ${ }^{9}$ when the intervention was also cost-neutral when considering health and care services used by both family carers and patients. ${ }^{9}$ To the best of our knowledge, there are no clinically and cost-effective interventions that have demonstrated effects beyond 5 years, ${ }^{12-14}$ and none are manualised; so the intervention can be delivered consistently to participants; by graduates without clinical training, with potential to implement at scale.

\section{Objectives}

Our aim was to determine the long-term (up to 6 years from baseline) clinical effectiveness of START for family carers' affective symptoms and costs compared with treatment as usual (TAU) in terms of:

(1) our primary outcome - Hospital Anxiety and Depression Scale - total score (HADS-T) $)^{15,16}$ in carers of people with dementia;

(2) secondary outcomes were:

(a) anxiety and depression caseness and scores,

(b) time until care home admission and death of the person with dementia, 
(c) time spent at home,

(d) cost of care for both people with dementia and carers.

\section{Method}

We registered a trial protocol before recruitment began at https:// doi.org/10.1186/ISRCTN70017938. After recruitment, the research team (with approval from the funding body while the database was still locked) agreed that the primary outcome should be changed to the total score on HADS as this has been shown to have better sensitivity and positive predictive value than either of the individual anxiety and depression scores in identifying depression and registered this prior to analyses. A standard reporting protocol was used.

After registration we requested and received a 5-year no-cost extension to the trial.

\section{Intervention and delivery}

We recruited 260 participants to the study. We developed the eightsession START manual-based individual coping intervention for dementia family carers from the American 'Coping with Caregiving. ${ }^{17}$ We trained and supervised non-clinically trained psychology graduates to deliver the intervention (see supplementary Fig. 1, available at https://doi.org/10.1192/bjp.2019.160), and P.R. supervised them clinically as a group with additional time available for individual support. There was a strong practical focus in the training programme on how to deliver the therapy, potential clinical dilemmas, empathic listening, effective use of supervision, safe working practise and when to ask for help. They were trained to adhere to the manual and we used role-play with senior members of the team completing a competency checklist to ensure they could deliver each session competently. We monitored intervention fidelity using a checklist out of a possible five points, and it was satisfactory. Therapists worked with carers to identify individual difficulties and to find workable solutions rather than give answers or recommendations, and implement strategies including: behavioural management, communication strategies, identifying and changing unhelpful thoughts, positive reframing, accessing support, future planning and increasing pleasant events. Each session included a relaxation exercise and we asked carers to put into practise the individualised strategies and relaxation between sessions. The final session was used to agree a plan of what to do in the future based upon what that carer had felt worked. The carer kept their own manual and relaxation compact discs.

In summary START is a parallel-group, superiority, singleblind, RCT conducted in the UK (four sites). Participants were selected to represent varied clinical services so we could see if the intervention was generalisable - a mental health trust based in a large city; a trust in a semi-rural area, a tertiary neurological clinic for rare and young-onset dementia; and a mental health trust where patients were allocated to a specialist nurse (Admiral nurse). We recruited self-identified family carers providing at least weekly support to people with a clinical diagnosis of dementia, living in their own homes and referred to the service we recruited from during the previous year. We excluded those who were unable to give informed consent or who lived more than $1.5 \mathrm{~h}$ travelling time from the researchers' base. We recruited from 4 November 2009 to 8 June 2011 through three mental health trusts and a tertiary neurology clinic. Last follow-up was 28 April 2017. Standard treatment includes medical, psychological and social interventions, consisting of assessment, diagnosis and information-giving, risk assessment and management (for example fire, driving, adequate nutrition and self-care, vulnerability, managing money), drug treatment, cognitive stimulation therapy, practical support, treatment of neuropsychiatric and cognitive symptoms, assessment of capacity, help in making long-term decisions and carer support. Patients in both groups received TAU and the use of services in both groups has been described in detail. ${ }^{18}$

\section{Randomisation and masking}

Participants were randomised 2:1 to intervention:TAU in order to maintain study power given the potential for clustering of outcomes by therapist in the intervention arm. Randomisation was stratified by centre using random permuted blocks via an online computergenerated randomisation system from an independent clinical trials unit. Assessors were masked to randomisation status, but study participants knew their allocation.

\section{Outcome measures}

We collected carer and patient sociodemographic details at baseline and measured dementia severity using the clinical dementia rating. ${ }^{19}$ We also administered the Neuropsychiatric Inventory (NPI) ${ }^{20}$ as neuropsychiatric symptoms have been shown to be associated with carer psychological morbidity, and the Zarit Burden Interview. ${ }^{21}$ Each NPI item is scored as the product of severity and frequency giving a potential score of $0-12$ and scores are summed giving a possible total from 0 to 144 . Higher scores indicate more neuropsychiatric symptoms and more burden, respectively.

We also measured carers' anxiety and depressive symptoms, using the HADS ${ }^{15,16}$ at baseline, 4, 8, 12 and 24 months. In an agreed extension with our funders and ethics committees we continued to collect carer HADS scores and place of residence for patients 6 monthly from 24 until 72 months. We recorded the date that a patient was admitted to a care home or had died, and stopped measuring the HADS at that point. HADS is a scale, validated for all age groups and settings, in people who are physically well or ill, and in Asian and African ethnic groups; ${ }^{21}$ summarised as HADS-D (depression) HADS-A (anxiety) with scores from 0 to 21 and a total HADS score (HADS-T) from 0 to 42 (higher scores indicating more symptoms). The total score (HADS-T) is our chosen primary outcome as it has better sensitivity and positive predictive value than either of the individual scales in identifying depression, when compared with ICD depression diagnosis criteria. ${ }^{16}$ HADS-D and HADS-A are also validated as scores for 'caseness' and were dichotomised as 'case' and 'non-case', with a cut-point of $\geq 9$. $^{21}$

The Client Service Receipt Inventory (CSRI) ${ }^{22}$ measured health and social care service use retrospectively until 24 months, but not beyond that point. Each carer reported their own and the patient's service use over the previous 4 months, covering the full range of services. ${ }^{8}$ Service contacts were multiplied by their unit costs (2009-2010 prices) obtained from publicly available sources: National Health Service (NHS) reference costs ${ }^{23}$ for in-patient and out-patient attendances, and the Personal Social Services Research Unit volume ${ }^{24}$ for other services. Costs were discounted to present values at an annual rate of $3.5 \%{ }^{25}$

Beyond the 24-month point, we estimated costs of services used by patients and carers up to the earliest of either withdrawal from the study, death of either patient or carer, or end of follow-up period (72 months). For patients who continued living in the community, we assumed that weekly costs remained the same as at 24 months. For patients moving to a care home, we attached a unit cost equal to the weekly charge for a private nursing home for older people, ${ }^{24}$ the most likely type of admission for someone with dementia, for the duration of stay, and we assumed that carer service use costs continued. Costs were carried forward as long as the patient/carer remained alive. 


\section{Statistical analysis}

Analyses were conducted as intention-to-treat based on a predefined statistical analysis plan. Most analyses were carried out in Stata (version 14) for Windows, but some models (as detailed) were fitted using $\mathrm{R}$.

\section{HADS scores}

HADS data included in the primary 72-month analysis are those collected while the carer was still actively looking after the patient (i.e. patient was still living at home). Data collected after the patient had died or was admitted to a care home were excluded.

To be included in the primary long-term analysis, the individual must have had at least one follow-up HADS-T score. Those excluded therefore have no follow-up measurements at any time point. Analyses compare the group as randomised, regardless of the number of therapy sessions attended in the intervention group

We used mixed-effects linear regression models to assess the effect of the START intervention on repeated measurements of HADS-T over 72 months. Initially we adjusted for treatment centre, HADS-T at baseline and time, but then extended this model to include adjustments for carer age, carer gender, baseline NPI score and Zarit score. We also investigated whether the treatment effect changed over time by including a treatment $\times$ time interaction. We chose not to allow for therapist clustering in these models as previous analyses of data up to 24 months had indicated that clustering effects were negligible. As a sensitivity analysis, however, models were refitted allowing for therapist clustering. For all cases estimates obtained were not substantially different.

We used scatter plots of residuals and fitted values to check model assumptions. The correlation structure assumed in the main analyses was compound symmetry; however, models were refitted in sensitivity analyses with alternative structures (autoregressive (order 1) and linear spatial correlation assumptions). For all models these investigations supported the models used for the main analyses.

The analyses described for the HADS-T were repeated for anxiety and depression subscales of the HADS. We investigated the effect of the START intervention on the occurrence of individuals with anxiety/depression, using mixed-effects logistic regression models, with a participant-level random effect.

If care home admission or death of the care recipient occurred prior to 72 months, the carer was not followed-up beyond the last visit prior to death or care home admission. Given the possibility of a relationship between HADS scores and death/care home admission, we conducted sensitivity analyses to consider the impact of such informative censoring. Joint mixed-effect models for the longitudinal HADS scores and time to admission to a home or death were fitted to account for the correlation between the longitudinal and survival outcomes. ${ }^{26}$ The HADS component treatment effect estimates were compared with those obtained from the previously fitted mixed models. (Joint models were fitted using the JM package in $\mathrm{R}^{27}$ ).

\section{Time until care home admission}

We employed a multistate model (depicted pictorially in supplementary Fig. 2$)^{28}$ to analyse time until care home admission while accounting for the possibility of patient death. The model was set up to allow transition from living at home to one of two states, care home admission or death. Effect estimates from the model are 'intensity ratios' that are analogous to hazard ratio estimates in a Cox proportional hazards model but pertain to the specific transitions within the multistate model. As before, models were fitted adjusting for centre, carer age, carer gender, baseline NPI and Zarit score. (Multistate models were fitted using the msm package in $\mathrm{R}^{28}$ ).
Time spent at home

In a further analysis of patient time spent at home (i.e. time prior to care home admission or death), we fitted models for time to admission or death using a standard survival analysis. We used a log rank test to make a comparison between the randomised groups and then fitted a Cox regression model to provide a treatment effect estimate adjusted for centre, carer age, carer gender, baseline HADS total, baseline NPI total and Zarit total score.

\section{Costs}

The difference in costs between treatment arms at 72 months was assessed using the non-parametric Wilcoxon Rank-Sum test. ${ }^{29}$

\section{Patient involvement}

This study was devised and conducted with patient and public involvement (PPI) in the questions of the study and PPI representatives were on the management and steering group. They helped shape the original questions, added qualitative questions about the experience and took part in interpreting the findings. They have also presented them.

\section{Results}

\section{Participant flow and recruitment}

The Consort diagram (Fig. 1) shows participant flow through the study. We randomised 260/472 (55\%) of the carers referred. Others refused $(n=181 ; 38 \%)$, did not meet inclusion criteria $(n=22 ; 5 \%)$ or were uncontactable $(n=9,2 \%)$. We randomised $173(67 \%)$ participants to intervention and 87 (33\%) to TAU. The characteristics of the randomised groups generally achieved good balance in terms of sociodemographic and clinical characteristics (see supplementary Table 1). Carers were mostly spouses/partners $(109 ; 42 \%)$ or children $(113 ; 44 \%)$. The proportions of patients who died (before they were admitted to a care home), were admitted to care homes and withdrew by randomised group is shown in supplementary Table 2. There is no evidence of significant differences in the proportions of participants in each end-status category between the START and TAU groups.

\section{Intervention adherence and fidelity}

In total, $130(75 \%)$ carers in the intervention group attended $\geq 5$ therapy sessions, 8 (5\%) withdrew before any therapy sessions. Ten therapists delivered the intervention, to between 11 and 32 carers each. The mean fidelity score was 4.7 (s.d. $=0.66$ )

\section{Primary outcome}

Table 1 summarises HADS-T scores at each follow-up point. Analysis of HADS-T, adjusting for centre, baseline score, time and factors related to outcome (carer age and gender, NPI, Zarit) over the 6-year period, showed an average improvement in HADS-T of 2.00 points compared with TAU (95\% CI -3.38 to $-0.63 ; P=0.005$ ) (Table 2). In the model adjusting only for centre, baseline score and time, average score decrease was smaller but still significant and in favour of the intervention group (Table 2). A model including an interaction with time showed no evidence of differential effects of the intervention over time $(P=0.98)$. 


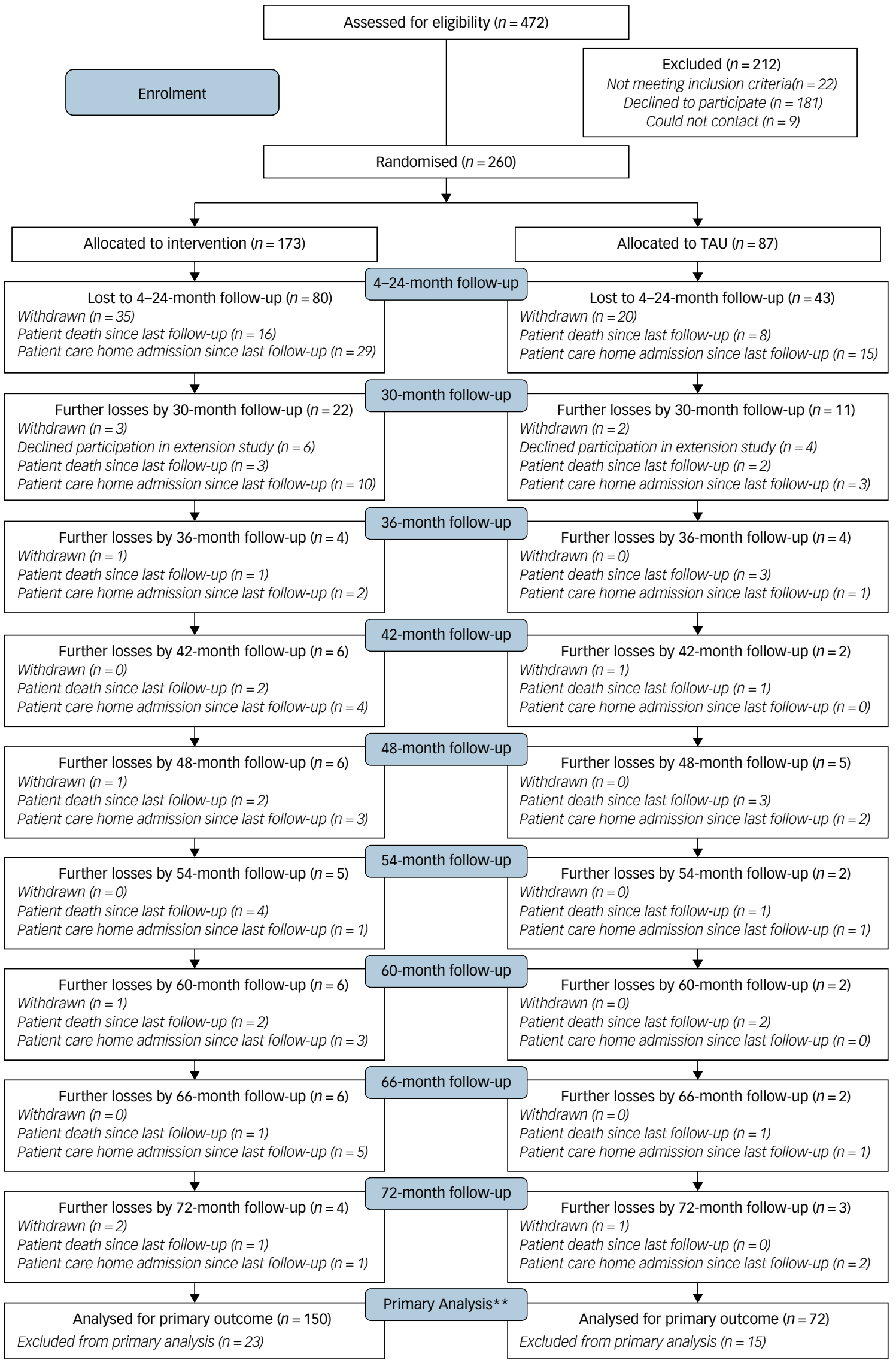

Fig. 1 CONSORT diagram for long-term outcomes (up to 72 months).

Hospital Anxiety and Depression Scale (HADS) data included in the primary 72-month analysis are collected while the carer was still looking after the patient at home. Prior to 24 months, carers were followed-up for HADS even after the patient had died or had been admitted to a care home (see Livingston et al. ${ }^{9}$ for more details). After 24 months, follow-up was terminated when the patient died or was no longer at home. For the purposes of the 6-year follow-up analysis, observation of HADS has been censored for all patients if either death or care home admission occurred. a. To be included in the primary long-term analysis, the individual must have at least one follow-up score available for the HADS total. TAU, treatment as usual. 
Table 1 Summaries of Hospital Anxiety and Depression Scale (HADS) total score at each follow-up time by treatment group

\begin{tabular}{|c|c|c|}
\hline $\begin{array}{l}\text { Follow-up } \\
\text { time (months) and group }\end{array}$ & $\begin{array}{l}\text { Number of } \\
\text { observations (n) }\end{array}$ & Mean (s.d.) \\
\hline \multicolumn{3}{|l|}{0} \\
\hline TAU & 87 & $14.8(7.4)$ \\
\hline START & 172 & $13.5(7.3)$ \\
\hline \multicolumn{3}{|l|}{4} \\
\hline TAU & 70 & $14.3(7.6)$ \\
\hline START & 146 & $12.3(7.3)$ \\
\hline \multicolumn{3}{|l|}{8} \\
\hline TAU & 67 & $14.9(8.1)$ \\
\hline START & 125 & $12.8(7.9)$ \\
\hline \multicolumn{3}{|l|}{12} \\
\hline TAU & 57 & $15.1(9.0)$ \\
\hline START & 122 & $12.5(7.8)$ \\
\hline \multicolumn{3}{|l|}{24} \\
\hline TAU & 44 & $15.6(8.7)$ \\
\hline START & 93 & $12.7(7.2)$ \\
\hline \multicolumn{3}{|l|}{30} \\
\hline TAU & 33 & $15.5(7.8)$ \\
\hline START & 65 & $13.0(7.5)$ \\
\hline \multicolumn{3}{|l|}{36} \\
\hline TAU & 28 & $15.6(7.5)$ \\
\hline START & 65 & $12.3(7.3)$ \\
\hline \multicolumn{3}{|l|}{42} \\
\hline TAU & 27 & $15.7(8.7)$ \\
\hline START & 59 & $13.8(8.0)$ \\
\hline \multicolumn{3}{|l|}{48} \\
\hline TAU & 22 & $16.5(8.9)$ \\
\hline START & 54 & $13.2(7.3)$ \\
\hline \multicolumn{3}{|l|}{54} \\
\hline TAU & 20 & $16.2(7.6)$ \\
\hline START & 49 & $12.1(7.0)$ \\
\hline \multicolumn{3}{|l|}{60} \\
\hline TAU & 18 & $17.3(10.3)$ \\
\hline START & 44 & $12.3(8.0)$ \\
\hline \multicolumn{3}{|l|}{66} \\
\hline TAU & 15 & 15.1 (9.5) \\
\hline START & 38 & $13(7.9)$ \\
\hline \multicolumn{3}{|l|}{72} \\
\hline TAU & 13 & 17.5 (11.1) \\
\hline START & 34 & $12.5(9.0)$ \\
\hline
\end{tabular}

\section{Secondary outcomes}

Depression and anxiety caseness and scores

In the fully adjusted analyses there was a reduced odds of HADSdepression caseness in the intervention group compared with TAU, (odds ratio $(\mathrm{OR})=0.20,95 \%$ CI $0.08-0.52, P=0.001$ ). Reduction in HADS-anxiety caseness, however, was not significant (OR $=0.50$, 95\% CI: 0.24-1.07, $P=0.07$ ) (Table 2).

Fully adjusted models for HADS-A and HADS-D continuous scores indicated significant beneficial intervention effects over
6 years, with average decreases of -0.97 (95\% CI -1.78 to -0.15 ) and -1.06 ( $95 \%$ CI -1.78 to -0.35$)$, respectively. Models showed no evidence of differential intervention effects with time for HADS-A or HADS-D ( $P=0.98$ and $P=0.94$, respectively).

Adjusted joint models were used as sensitivity analyses to allow for the possibility of a relationship between HADS scores and time to care home admission or death and these gave similar results to previous models for HADS-T, HADS-D and HADS-A (HADS-T: 2.01 ( $95 \%$ CI -3.38 to -0.63 ), HADS-D: -1.07 ( -1.78 to -0.37 ), HADS-A: -0.97 ( -1.78 to -0.16$)$ ). This suggests that censoring by death/care home admission is not problematic.

\section{Analysis of time until patient care home admission and death}

Figure 2 shows the cumulative incidence of care home admission and death over time by randomised group and indicates little difference between the groups for either outcome. The multistate model adjusted for centre, carer age, carer gender, baseline HADS-T, baseline NPI and baseline Zarit gave intensity ratios for the START intervention versus TAU of 0.88 (95\% CI $0.58-1.35)$ for the home-to-care-home transition and 0.81 (95\% CI 0.50-1.30) for the home-to-death transition, both showing no evidence of a between-group difference in transition rates to care homes or in death rates.

\section{Analysis of time spent at home}

Based on Kaplan Meier estimates, the estimated median time spent at home (i.e. time until death or admission to a care home) for the TAU group was 39.0 months (95\% CI 31.1-49.4) and for START was 42.2 months (95\% CI 33.3-54.7). Cox regression with adjustments for centre, carer age, baseline HADS total, NPI score and Zarit score, showed no evidence of a difference between the randomised groups (hazard ratio estimate: 0.81 (95\% CI 0.59-1.11)).

Costs

Costs for carer and patient service use are shown in Table 3. Costs of services used by patients were much higher than costs for services used by carers across the full study period. In the final year of follow-up (61-72 months) median patient service use costs were $£ 16964$ for TAU and $£ 5759$ for START $(P=0.072)$. Median carer service use costs were $£ 377$ for TAU and $£ 274$ for START.

\section{Discussion}

\section{Main findings}

This is the first RCT to demonstrate that family carers of people with dementia referred to specialist care experience benefits from an intervention delivered by supervised psychology graduates in terms of depression and anxiety symptoms and depression caseness,

\begin{tabular}{|c|c|c|}
\hline \multirow[b]{2}{*}{ HADS measure } & \multicolumn{2}{|c|}{ Estimates comparing intervention and TAU } \\
\hline & $\begin{array}{l}\text { Adjusted for centre, baseline } \\
\text { score and time }(n=222)\end{array}$ & $\begin{array}{l}\text { Adjusted for centre, baseline score, } \\
\text { time, age, gender, NPI \& Zarit }(n=213)\end{array}$ \\
\hline \multicolumn{3}{|c|}{ Difference in means $(95 \% \mathrm{Cl})$} \\
\hline HADS-T (total score) & $-1.45(-2.80$ to -0.10$)$ & $-2.00(-3.38$ to -0.63$)$ \\
\hline HADS-D & $-0.93(-1.63$ to -0.24$)$ & $-1.06(-1.78$ to -0.35$)$ \\
\hline HADS-A & $-0.58(-1.39$ to 0.22$)$ & $-0.97(-1.78$ to -0.15$)$ \\
\hline \multicolumn{3}{|l|}{ Odds ratio $(95 \% \mathrm{Cl})$} \\
\hline HADS-D caseness & $0.30(0.13-0.71)$ & $0.20(0.08-0.52)$ \\
\hline HADS-A caseness & $0.64(0.31-1.32)$ & $0.50(0.24-1.07)$ \\
\hline
\end{tabular}




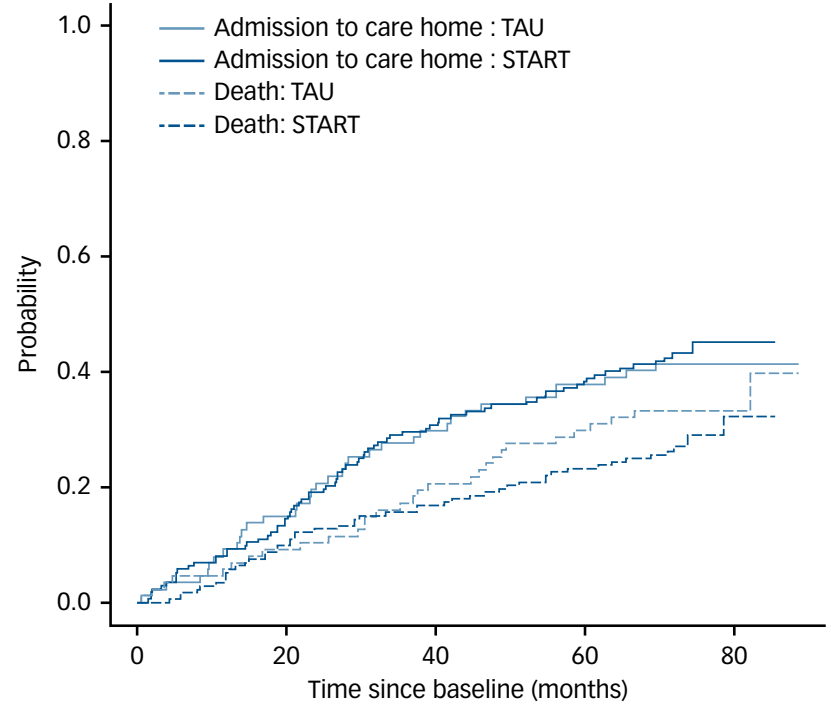

Fig. 2 Plot of estimated cumulative incidence functions for the events 'care home admission' and 'death' over time, stratified by treatment group.

TAU, treatment as usual; START, STrAtegies for RelaTives.

not only in the short term but for up to 6 years. The difference is small but is statistically significant, greater than the minimally clinically important difference (that which is clinically significant to patients) and is sustained. ${ }^{30}$ The difference in costs appears to be economically large (cost per patient in the intervention group is around a third of the cost in the TAU group) although there was no significant difference in time to care home admission or death. The reduced sample size, however, means that the test for differences in cost is underpowered (particularly given highly skewed cost data), but the estimated costs of health and care services used by patients appear to be lower for the intervention group compared with TAU in the final year of follow-up. It is encouraging that this intervention does not therefore increase costs, and might actually be cost-saving. Carers in the control group were five times more likely to have clinically significant depression on a rating scale validated against caseness using ICD criteria. Predictably, health and social care costs increase over time for both groups, as a result of the worsening condition. There is a bigger increase in TAU group.

\section{Strengths and limitations}

The trial is randomised, with masked follow-ups and we recruited the numbers of participants needed according to the power analysis based on the primary clinical outcome. ${ }^{7}$ The intervention was manual-based, standardised and supervised. High fidelity ratings and very low intercluster correlations show the results do not differ according to therapists, suggesting that the intervention can be delivered consistently.

We planned a pragmatic trial to include all family carers who presented to services so they had varied sociodemographic and clinical characteristics and came from a variety of services; consequently, our study has some external generalisability, that is, it suggests the intervention can be used in a variety of NHS settings. We did not have the power to analyse whether this intervention was more effective in subgroups; for example, those with more education or without a mental health history or with more family support. At the time of the START intervention most patients had only recently presented to services and thus the intervention can be offered at the beginning of the patient pathway but may not be applicable to those who have had the diagnosis for many years. It was preventative as well as improving depression and so can be offered to those with and without depression. ${ }^{7}$ Previously we have found that carers used different components of the intervention and some continued to use these consciously over 2 years but we did not ask about this at 6-year follow-up. ${ }^{11}$ Only patient care home admission and death and carer HADS were directly collected after 2 years and therefore the economic analysis involved modelling. Although the differences in costs were striking, the nature of dementia, which inevitably meant attrition by death of some of those with it over 6 years, meant the numbers were smaller. In addition, the data were skewed and they only approached the usual level taken as significant.

\section{Comparison with other studies, meaning and implications}

The practical nature of the intervention, in which carers were encouraged to develop and continue to use successful strategies, might also account for the longevity of the positive effects on carer mental health that we found - the most successful strategies were likely to be used repeatedly and therefore remembered and integrated into caring routines. The intervention included a final session on planning for the future. It is likely that the nature of caring difficulties will have evolved over 6 years. Intervention group participants were given a manual in which strategies they had found helpful for managing caring challenges, as well as pleasant events, were recorded and it

\begin{tabular}{|c|c|c|c|c|c|c|}
\hline \multirow[b]{2}{*}{ Time period and services for patient or carer } & \multicolumn{3}{|c|}{ Intervention } & \multicolumn{3}{|c|}{ Treatment as usual } \\
\hline & $n$ & Median & Interquartile range & $n$ & Median & Interquartile range \\
\hline \multicolumn{7}{|l|}{$25-36$ months } \\
\hline Carer & 82 & 364 & $132-704$ & 35 & 269 & $103-622$ \\
\hline Patient & 109 & 5764 & $1922-18869$ & 54 & 5303 & $1573-21866$ \\
\hline \multicolumn{7}{|l|}{ 37-48 months } \\
\hline Carer & 83 & 402 & 130-702 & 35 & 279 & $166-601$ \\
\hline Patient & 94 & 6098 & 1767-20 219 & 44 & 7200 & $1452-22346$ \\
\hline \multicolumn{7}{|l|}{ 49-60 months } \\
\hline Carer & 73 & 390 & $137-666$ & 28 & 274 & 178-587 \\
\hline Patient & 83 & 4619 & $1744-23116$ & 33 & 16574 & 1524-24920 \\
\hline \multicolumn{7}{|l|}{ 61-72 months } \\
\hline Carer & 53 & 377 & 184-635 & 24 & 274 & 191-587 \\
\hline Patient & 68 & 5759 & $1892-18254$ & 30 & 16964 & 2369-24 077 \\
\hline
\end{tabular}


also included recordings of the relaxation exercises so that they had something to refer to during future caring.

Our findings would suggest that carers were able to continue using the skills and strategies they had practised in the longer term; a focus on planning for the future, accessing support and explicit consideration of how difficulties may change and emotion-focused and acceptance-based strategies, might have helped support this. It is also possible that carers revisited previously less personally relevant aspects of the manualised intervention as certain issues or challenges became more salient to their caring.

Many interventions for family carers of people with dementia have not worked in improving mood. ${ }^{31-33}$ Others have been effective but the effects have not been sustained. ${ }^{34}$ Most have not considered prevention. In general, those that have been effective are multicomponent and delivered to individuals rather than groups for at least six sessions ${ }^{35,36}$ and our study was designed to follow this model. Some earlier interventions for family carers have been effective and had sustained effects that have continued for between 1 and 5 years. ${ }^{12-14}$ Our study is in line with this but because it is manual-based and delivered by non-clinically trained psychology graduates it is designed to be scalable and practical and has economic findings to support this. We have more fully considered cost than most other studies although there is some evidence that interventions can generate saving. ${ }^{37,38}$ There is little evidence that carer stress predicts care home admission in community-dwelling older people in general ${ }^{39}$ but psychological interventions for family carers may reduce care home admission for people living with dementia, with a meta-analysis of the best-quality studies finding a significant reduction in the odds of care home admission, although the time to admission difference did not reach significance. ${ }^{40}$ Family carers become more anxious and depressed over time without intervention; thus we included carers who were not depressed at presentation. ${ }^{3,4}$

\section{Future research}

The START intervention is clinically effective, improving carer mood over 6 years. It does not increase patient or carer servicerelated costs and thus should be made available. The numbers of people with dementia and the diversity of culture, geographic location and available NHS resources mean that further research is necessary to widen access and optimise implementation. For example, to consider whether the intervention can be delivered remotely (through a skype or similar application), through the existing voluntary sector carer support infrastructure (as some carers do not see themselves as patients) and be adapted for ethnic groups with different cultures.

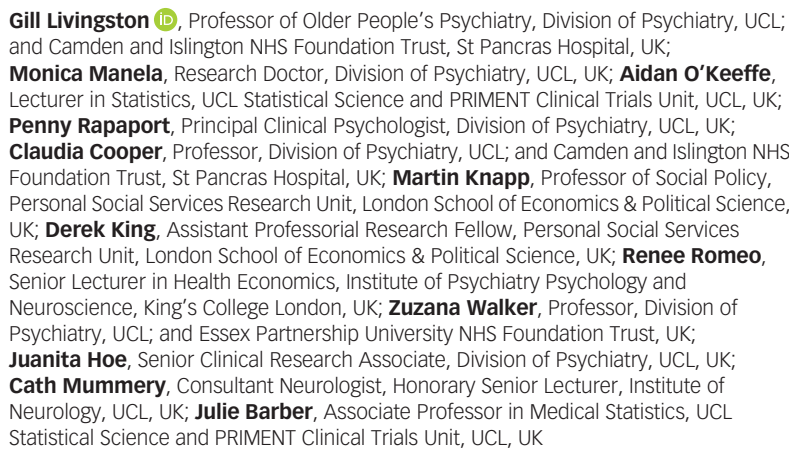

Correspondence: Gill Livingston, UCL, Division of Psychiatry, Floor 6, Maple house, 149 Tottenham Court Rd, W1T 7NF, UK. Email: g.livingston@ucl.ac.uk

First received 11 Jul 2018, final revision 7 May 2019, accepted 1 Jun 2019

\section{Supplementary material}

Supplementary material is available online at https://doi.org/10.1192/bjp.2019.160.

\section{Funding}

This project was funded by the National Institute for Health Research Health Technology Assessment (NIHR HTA) Programme (project number 08/14/06). The authors analysed results and prepared this manuscript independently of the funding body.

The views and opinions expressed therein are those of the authors and do not necessarily reflect those of the HTA programme, NIHR, NHS or the Department of Health. The study was sponsored by UCL. Neither funders nor sponsors had a role in the study design and the collection, analysis, and interpretation of data and the writing of the article and the decision to submit it for publication. The researchers were independent from funders and sponsors. All researchers could access all the data. All authors, external and internal, had full access to all of the data (including statistical reports and tables) in the study and can take responsibility for the integrity of the data and the accuracy of the data analysis.

\section{References}

1 Mahoney R, Regan C, Katona C, Livingston G. Anxiety and depression in family caregivers of people with Alzheimer disease: the LASER-AD study. Am J Geriatr Psychiatry 2005; 13: 795-801.

2 Cooper C, Balamurali TB, Livingston G. A systematic review of the prevalence and covariates of anxiety in caregivers of people with dementia. Int Psychogeriatr 2007; 19: 175-95.

3 Goren A, Montgomery W, Kahle-Wrobleski K, Nakamura T, Ueda K. Impact of caring for persons with Alzheimer's disease or dementia on caregivers' health outcomes: findings from a community based survey in Japan. BMC Geriatr 2016; 16: 122

4 Livingston G, Sommerlad A, Orgeta V, Costafreda SG, Huntley J, Ames D, et al. Dementia prevention, intervention, and care. Lancet 2017; 390: 2673-734.

5 Prince M, Wimo A, Guerchet M, Ali G, Wu YT, Prina M. World Alzheimer Report 2015 - The Global Impact of Dementia: An Analysis of Prevalence, Incidence, Cost and Trends. Alzheimer's Disease International (ADI), London, 2015.

6 Winblad B, Amouyel P, Andrieu S, Ballard C, Brayne C, Brodaty H, et al. Defeating Alzheimer's disease and other dementias: a priority for European science and society. Lancet Neurol 2016; 15: 455-532.

7 Livingston G, Barber J, Rapaport P, Knapp M, Griffin M, King D, et al. Clinical effectiveness of a manual based coping strategy programme (START, STrAtegies for RelaTives) in promoting the mental health of carers of family members with dementia: pragmatic randomised controlled trial. BMJ 2013; 347: $\$ 6276$

8 Knapp M, King D, Romeo R, Schehl B, Barber J, Griffin M, et al. Cost effectiveness of a manual based coping strategy programme in promoting the mental health of family carers of people with dementia (the START (STrAtegies for RelaTives) study): a pragmatic randomised controlled trial. Br Med J 2013; 347: f6342.

9 Livingston G, Barber J, Rapaport P, Knapp M, Griffin M, King D, et al. Long-term clinical and cost-effectiveness of psychological intervention for family carers of people with dementia: a single-blind, randomised, controlled trial. Lancet Psychiatry 2014; 1: 539-48.

10 Li R, Cooper C, Barber J, Rapaport P, Griffin M, Livingston G. Coping strategies as mediators of the effect of the START (strategies for RelaTives) intervention on psychological morbidity for family carers of people with dementia in a randomised controlled trial. J Affect Disord 2014; 168C: 298-305.

11 Sommerlad A, Manela M, Cooper C, Rapaport P, Livingston G. START (STrAtegies for RelaTives) coping strategy for family carers of adults with dementia: qualitative study of participants' views about the intervention. $B M J$ Open 2014; 4: e005273.

12 Sorensen S, Duberstein P, Gill D, Pinquart M. Dementia care: mental health effects, intervention strategies, and clinical implications. Lancet Neurol 2006; 5: $961-73$

13 Mittelman MS, Roth DL, Coon DW, Haley WE. Sustained benefit of supportive intervention for depressive symptoms in caregivers of patients with Alzheimer's disease. Am J Psychiatry 2004; 161: 850-6.

14 Mittelman MS, Roth DL, Clay OJ, Haley WE. Preserving health of Alzheimer caregivers: impact of a spouse caregiver intervention. Am J Geriatr Psychiatry 2007; 15: 780-9.

15 Zigmond AS, Snaith RP. The Hospital Anxiety and Depression Scale. Acta Psychiatr Scand 1983; 67: 361-70.

16 Spinhoven P, Ormel J, Sloekers PPA, Kempen GIJM, Speckens AEM VanHemert AM. A validation study of the Hospital Anxiety and Depression Scale (HADS) in different groups of Dutch subjects. Psychol Med 1997; 27: 363-70. 
17 Gallagher-Thompson D, Solano N, McGee JS, Krisztal E, Kaye J, Coon DW, et al. Coping with Caregiving: Reducing Stress and Improving Your Quality of Life. Stanford University School of Medicine and VA Palo Alto Healthcare System, 2002.

18 Knapp M, King D, Romeo R, Schehl B, Barber J, Griffin M, et al. Cost effectiveness of a manual based coping strategy programme in promoting the mental health of family carers of people with dementia (the START (STrAtegies for RelaTives) study): a pragmatic randomised controlled trial. Br Med J 2013; 347: f6342.

19 Morris JC. Clinical dementia rating: a reliable and valid diagnostic and staging measure for dementia of the Alzheimer type. Int Psychogeriatr 1997; 9 (Suppl 1): 173-6.

20 Cummings JL, Mega M, Gray K, Rosenberg-Thompson S, Carusi DA, Gornbein J. The Neuropsychiatric Inventory: comprehensive assessment of psychopathology in dementia. Neurology 1994; 44: 2308-14.

21 Bjelland I, Dahl AA, Haug TT, Neckelmann D. The validity of the Hospital Anxiety and Depression Scale. An updated literature review. J Psychosom Res 2002: 52: 69-77.

22 Beecham J, Knapp M. Costing psychiatric intervention. In Measuring Mental Health Needs (eds C Thornicroft, C Brewin, J Wing), Gaskell, 1992.

23 Coon DW, Thompson L, Steffen A, Sorocco K, Gallagher-Thompson D. Anger and depression management: psychoeducational skill training interventions for women caregivers of a relative with dementia. Gerontologist 2003; 43: 678-89.

24 Curtis L. The Unit Costs of Health and Social Care 2010-2011. DHSC, 2011. (www.dh.gov.uk/en/Publicationsandstatistics/Publications/Publications PolicyAndGuidance/DH_123459).

25 National Institute for Health and Care Excellence. Guide to the Methods of Technology Appraisal. NICE, 2013

26 Henderson R, Diggle P, Dobson A. Joint modelling of longitudinal measurements and event time data. Biostatistics 2000; 1: 465-80.

27 Rizopoulos D. JM:An R package for the joint modelling of longitudinal and timeto-event data. J Stat Software 35: 9

28 Meira-Machado L, de Una-Alvarez J, Cadarso-Suarez C, Andersen PK. Multistate models for the analysis of time-to-event data. Stat Methods Med Res 2009; 18: 195-222.

29 Wilcoxon F. Individual comparisons of grouped data by ranking methods. J Econ Entomol 1946; 39: 269.

30 Puhan MA, Frey M, Buchi S, Schunemann HJ. The minimal important difference of the Hospital Anxiety and Depression Scale in patients with chronic obstructive pulmonary disease. Health Qual Life Outcomes 2008; 6: 46.
31 Farran CJ, Paun O, Cothran F, Etkin CD, Rajan KB, Eisenstein A, et al. Impact of an individualized physical activity intervention on improving mental health outcomes in family caregivers of persons with dementia: a randomized controlled trial. AIMS Medical Science 2015; 3: 31

32 Charlesworth G, Shepstone L, Wilson E, Reynolds S, Mugford M, Price D, et al. Befriending carers of people with dementia: randomised controlled trial. BMJ 2008: 336: 1295-7.

33 Waldorff FB, Buss DV, Eckermann A, Rasmussen ML, Keiding N, Rishoj S, et al. Efficacy of psychosocial intervention in patients with mild Alzheimer's disease: the multicentre, rater blinded, randomised Danish Alzheimer Intervention Study (DAISY). BMJ 2012; 345: e4693.

34 Moore RC, Chattillion EA, Ceglowski J, Ho J, von Känel R, Mills PJ, et al. A randomized clinical trial of Behavioral Activation (BA) therapy for improving psychological and physical health in dementia caregivers: Results of the Pleasant Events Program (PEP). Behav Res Therapy 2013; 51: 623-32.

35 Selwood A, Johnston K, Katona C, Lyketsos C, Livingston G. Systematic review of the effect of psychological interventions on family caregivers of people with dementia. J Affect Disord 2007; 101: 75-89.

36 Dickinson C, Dow J, Gibson G, Hayes L, Robalino S, Robinson L. Psychosocial intervention for carers of people with dementia: what components are most effective and when? A systematic review of systematic reviews. Int Psychogeriatr 2017; 29: 31-43.

37 Knapp M, Lemmi V, Romeo R. Dementia care costs and outcomes: a systematic review. Int J Geriatr Psychiatry 2012; 28: 551-61.

38 Clarkson $\mathrm{P}$, Davies L, Jasper R, Loynes N, Challis D. A Systematic Review of the Economic Evidence for Home Support Interventions in Dementia. Value in Health, 2017.

39 Donnelly NA, Hickey A, Burns A, Murphy P, Doyle F. Systematic review and meta-analysis of the impact of carer stress on subsequent institutionalisation of community-dwelling older people. PLOS One 2015; 10: e0128213.

40 Spijker A, Vernooij-Dassen M, Vasse E, Adang E, Wollersheim H, Grol R, et al. Effectiveness of nonpharmacological interventions in delaying the institutionalization of patients with dementia: a meta-analysis. J Am Geriatr Soc 2008; 56: $1116-28$

\title{
100 words on reward prediction error
}

\author{
Karl Friston (iD
}

Reward prediction error is like Marmite - you either love it or hate it. I hate it because it commits to a view of the brain that inherits from 20th-century behaviourism and reinforcement learning. When people say dopamine encodes reward prediction error, they are assuming that the brain is in the game of maximising reward. But it is not-the brain updates its beliefs and selects a preferred course of action. On this (planning as active inference) view, the available evidence suggests that dopamine encodes the precision of beliefs about policies - or, more simply, the confidence afforded (subpersonal) plans of action. 